\title{
NEARLY CONCONCENTRIC KORTEWEG-DE VRIES EQUATION AND PERIODIC TRAVELING WAVE SOLUTION
}

\author{
YUNKAI CHEN \\ Department of Mathematics and Computer Science \\ Fayetteville State University \\ Fayetteville, North Carolina 28301-4298, USA
}

(Received March 8, 1996)

\begin{abstract}
The generalized nearly concentric Korteweg-de Vries equation $\left[u_{\eta}+u /(2 \eta)+u^{n} u_{\xi}+u_{\xi \xi \xi}\right]_{\xi}$ $+u_{\theta \theta} / \eta^{2}=0$ is considered. The author converts the equation into the power Kadomtsev-Petviashvili equation $\left[u_{t}+u^{n} u_{x}+u_{x x x}\right]_{x}+u_{y y}=0$. Solitary wave solutions and cnoidal wave solutions are obtained The cnoidal wave solutions are shown to be representable as infinite sums of solitons by using Fourier series expansions and Poisson's summation formula
\end{abstract}

KEY WORDS AND PHRASES: Korteweg-de Vries equation, Kadomtsev-Petviashvili equation, solitary wave solution, cnoidal wave solution.

AMS SUBJECT CLASSIFICATION CODES: 35B20, 35Q20, 76B15

\section{INTRODUCTION}

The concentric Korteweg-de Vries equation (also called cylindrical Korteweg-de Vries equation),

$$
u_{\eta}+u /(2 \eta)+u u_{\xi}+u_{\xi \xi \xi}=0,
$$

was first derived by Maxon and Viecelli in 1974 from the study of propagation of radically ingoing acoustic waves in cylindrical geometry [1]. In the equation, $u=u(\xi, \eta), \eta=\epsilon^{3 / 2} \omega_{2} t$, and $\xi=-\epsilon^{1 / 2}\left(r / \lambda_{D}+\omega_{2} t\right)$, where $\epsilon$ is the expansion parameter, $\lambda_{D}$ the Debye length, $\omega_{2}$ the ion plasma frequency, $r$ the radial distance, and $t$ the time.

As the one-dimensional Korteweg-de Vries equation ( $\mathrm{KdV}$ equation for short) can be extended to the two-dimensional $\mathrm{KdV}$ equation, so the concentric $\mathrm{KdV}$ equation can be generalized to some higher dimensional equations. Considering the nearly straight wave propagation which varies in a very small angular region, Johnson derived the following nearly concentric $\mathrm{KdV}$ equation which is one of the generalized equations from Eq. (1.1)

$$
\left[u_{\eta}+u /(2 \eta)+u u_{\xi}+u_{\xi \xi \xi}\right]_{\xi}+u_{\theta \theta} / \eta^{2}=0
$$

where $u=u(\eta, \xi, \theta)$ and $\theta$ is the angular variable which varies in a small region [2,3].

In this paper, the author considers the power nearly concentric $\mathrm{KdV}$ equation of the form

$$
\left[u_{\eta}+u /(2 \eta)+u^{n} u_{\xi}+u_{\xi \xi \xi}\right]_{\xi}+u_{\theta \theta} / \eta^{2}=0
$$

where $n$ is a positive integer. Eq. (1.3) is converted to the power Kadomtsev-Petviashvili equation (KP equation for short). Chen and Wen's method [4] is applied to the power KP equation to obtain ordinary differential equations The solitary wave solutions and cnoidal wave solutions can be expressed as sums 
of infinite number of solitons by using Fourier series expansions and Poisson's summation formula. The author has also established a criterion for the existence of a single soliton solution, it is $C>0$, where $C=\left(a \omega-b^{2}\right) / a^{2}($ see Section 3$)$.

\section{FORMULATION OF THE PROBLEM}

We start from the power nearly concentric KdV equation

$$
\left[u_{\eta}+u /(2 \eta)+u^{n} u_{\xi}+u_{\xi \xi \xi}\right]_{\xi}+u_{\theta \theta} / \eta^{2}=0,
$$

where $n$ is a positive integer. Eq. (2.1) reduces to the usual nearly concentric $\mathrm{KdV}$ equation when $n=1$ Considering that Eq. (2.1) is more analogous to the two-dimensional case and motivated by the results obtained by Chen and Wen [5] and Johnson [2,3,6], the author introduces the transformations $\eta=t$, $\xi=x+y^{2} /(4 t)$, and $\theta=y / t$. One can argue that since $\tan \theta=y / x$ and $\theta$ is the variable in a very small angular sector, $\theta$ can be used to approximate $y / x$. Thus when $x$ and $t$ are large and of the same order, it seems to be reasonable to assume $\theta=y / t$. Therefore, $u(\xi, \eta, \theta)=u(x, y, t)$, and

$$
\begin{gathered}
u_{t x}=u_{\eta \xi}-\frac{y^{2}}{4 \eta^{2}} u_{\xi \xi}, \quad\left(u^{n} u_{x}\right)_{x}=\left(u^{n} u_{\xi}\right)_{\xi}, \\
u_{x x x x}=u_{\xi \xi \xi \xi}, \quad u_{y y}=\frac{y^{2}}{4 \eta^{2}} u_{\xi \xi}+\frac{1}{2 \eta} u_{\xi}+\frac{1}{\eta^{2}} u_{\theta \theta} .
\end{gathered}
$$

Hence Eq. (2.1) can be converted to the power KP equation

$$
\left[u_{t}+u^{n} u_{x}+u_{x x x}\right]_{x}+u_{y y}=0,
$$

where $n$ is a positive integer [7].

We now look for the real-valued traveling wave solution of the form $U(z)=u(x, y, t)$ with $z=a x+b y-\omega t$, where $a, b$, and $\omega$ are real constants. Without loss of generality we assume $a>0$. Substitution into Eq. (2.2) yields the fourth order nonlinear ordinary differential equation of $U$

$$
-\left(a w-b^{2}\right) U^{\prime \prime}+a^{2}\left(U^{n} U^{\prime}\right)^{\prime}+a^{4} U^{(4)}=0 .
$$

Integrating Eq. (2.3) twice with respect to $z$ yields the second order equation:

$$
-\left(a w-b^{2}\right) U+\frac{a^{2}}{n+1} U^{n+1}+a^{4} U^{\prime \prime}=A z+B a^{2},
$$

where $A$ and $B$ are integration constants.

\section{SOLITARY WAVE SOLUTIONS}

For the existence of solitary wave solution, we impose the boundary conditions $U, U^{\prime}, U^{\prime \prime}, U^{\prime \prime \prime} \rightarrow 0$ when $z \rightarrow \pm \infty$. These conditions imply $A=B=0$ in Eq. (2.4). Using the fact $U^{\prime \prime}=d\left(U^{\prime}\right)^{2} /(2 d U)$, we can obtain from Eq. (2.4)

$$
\frac{1}{2} U^{\prime 2}=\frac{1}{a^{2}} U^{2}\left[\frac{C}{2}-\frac{U^{n}}{(n+1)(n+2)}\right],
$$

where $C=\left(a w-b^{2}\right) / a^{2}$. It can be verified that when $C \leq 0$, solutions to Eq. (3.1) exist only when $n$ is odd and these solutions are unbounded. Therefore, they are not of much physical interest. Eq. (3.1) has nontrivial solitary wave solution when $C>0$, and the solitary wave solution is

$$
U(z)=\left\{\frac{C(n+1)(n+2)}{2} \operatorname{sech}^{2}\left(\frac{n \sqrt{C}}{2 a}\left(z-z_{0}\right)\right)\right\}^{1 / n},
$$

where $z_{0}$ is an integration constant Since $\operatorname{sech}^{2} X=1 / \cosh ^{2} X=4 /\left(e^{X}+e^{-X}\right)^{2}$, the solitary wave described by the solution decays exponentially for $z \rightarrow \pm \infty$ Furthermore, we note that $C>0$ gives a 
condition under which a nontrivial solitary wave solution exists. This condition indicates a relationship that must be satisfied by the three constants $a, b$, and $\omega$, namely, $a w>b^{2}$. On the other hand, if $a \omega \leq b^{2}$, either no real solution exists or the solutions are unbounded.

\section{CNOIDAL WAVE SOLUTION WHEN $\boldsymbol{n}=1$}

For the existence of bounded solutions we assume $A=0$, and hence when $n=1$ we obtain from Eq (2 4)

$$
U^{\prime 2}=\frac{1}{3 a^{2}}\left(-U^{3}+3 C U^{2}+6 B U+D\right)=\frac{1}{3 a^{2}} F(U),
$$

where $C=\left(a \omega-b^{2}\right) / a^{2}, \quad D$ is an integration constant, and $F(U)$ is the cubic function $-U^{3}+3 C U^{2}+6 B U+D$.

For the existence of periodic traveling wave solution, the cubic function $F(U)$ in the right-hand side of Eq. (4 1) plays an important role. It is shown in Reference [4] that a cnoidal wave solution exists only if $F(U)$ has three distinct real simple zeros $U_{1}, U_{2}$, and $U_{3}$ such that $U_{3}<U_{2}<U_{1}$ and $U_{2} \leq U(z) \leq U_{1} \quad$ In this case, we can write Eq. (4.1) as the following by separating variables

$$
\begin{aligned}
\frac{1}{\sqrt{3} a}\left(z_{1}-z\right) & =\int_{U}^{U_{1}} \frac{d U}{\sqrt{F(U)}} \\
& =\int_{U}^{U_{1}} \frac{d U}{\sqrt{\left(U_{1}-U\right)\left(U-U_{2}\right)\left(U-U_{3}\right)}},
\end{aligned}
$$

where $z_{1}$ is a value at which $U\left(z_{1}\right)=U_{1}$. The period $T$ in $z$ is given by

$$
T=2 \sqrt{3} a \int_{U_{2}}^{U_{1}} \frac{d U}{\sqrt{\left(U_{1}-U\right)\left(U-U_{2}\right)\left(U-U_{3}\right)}} .
$$

Eq (4.2) can also be expressed in terms of elliptic and trigonometric functions as

$$
\frac{1}{\sqrt{3} a}\left(z_{1}-z\right)=\frac{2}{\sqrt{U_{1}-U_{3}}} s n^{-1}(\sin \phi, k)=\frac{2}{\sqrt{U_{1}-U_{3}}} F(\phi, k)
$$

where $\phi=\sin ^{-1} \sqrt{\left(U_{1}-U\right) /\left(U_{1}-U_{2}\right)}, k^{2}=\left(U_{1}-U_{2}\right) /\left(U_{1}-U_{3}\right)$, and $F(\phi, k)=s n^{-1}(\sin \phi, k)$ is the normal elliptic integral of the first kind with modulus $k$ [8].

Denote $F(\phi, k)$ by $v$, we then have

$$
\begin{gathered}
v=\frac{1}{2 \sqrt{3} a} \sqrt{U_{1}-U_{3}}\left(z_{1}-z\right), \\
s n(v, k)=\sin \phi=\sqrt{\left(U_{1}-U\right) /\left(U_{1}-U_{2}\right)},
\end{gathered}
$$

and hence the cnoidal wave solution is obtained

$$
\begin{aligned}
U(z) & =U_{1}-\left(U_{1}-U_{2}\right) s n^{2}(v, k) \\
& =U_{2}+\left(U_{1}-U_{2}\right) c n^{2}(v, k) \\
& =U_{3}+\left(U_{1}-U_{3}\right) d n^{2}(v, k) \\
& =U_{3}+\left(U_{1}-U_{3}\right) d n^{2}\left(\frac{1}{2 \sqrt{3} a} \sqrt{U_{1}-U_{3}}\left(z-z_{1}\right), k\right),
\end{aligned}
$$

where $c n(v, k)=\cos \phi$ and $d n(v)=,\sqrt{1-k^{2} \sin ^{2} \phi}$.

It should be noted that for the existence of cnoidal wave solution there is no restriction on $C$ which can be positive, zero or negative as long as $C=\frac{1}{3}\left(U_{1}+U_{2}+U_{3}\right)$.

Using the Fourier series expansion of $d n^{2}(v, k)$ [9] and the Poisson's summation formula [10], we have 


$$
d n^{2}(v, k)=\frac{E}{K}-\frac{\pi}{2 K K^{\prime}}+\frac{\pi^{2}}{4 K^{\prime 2}} \sum_{m=-\infty}^{\infty} \operatorname{sech}^{2}\left[\frac{\pi}{2 K^{\prime}}(v-2 m K)\right],
$$

where $K=\int_{0}^{\pi / 2} d \theta / \sqrt{1-k^{2} \sin ^{2} \theta}$ is the complete elliptic integral of the first kind with modulus $k$; $K^{\prime}=\int_{0}^{\pi / 2} d \theta / \sqrt{1-k^{\prime 2} \sin ^{2} \theta}$ is the complete elliptic integral of the first kind with modulus $k^{\prime}=\sqrt{1-k^{2}}$; and $E=\int_{0}^{\pi / 2} \sqrt{1-k^{2} \sin ^{2} \theta} d \theta$ is the complete elliptic integral of the second kind with modulus $k$. Therefore, the cnoidal wave solution $U(z)$ in Eq. (4.5) can be expressed as

$$
U(z)=P+Q \sum_{m=-\infty}^{\infty} \operatorname{sech}^{2} R\left(z-z_{1}+m T\right),
$$

where

$$
\begin{aligned}
& P=U_{3}+\left(U_{1}-U_{3}\right)\left[\frac{E}{K}-\frac{\pi}{2 K K^{\prime}}\right], \\
& Q=\left(U_{1}-U_{3}\right) \frac{\pi^{2}}{4 K^{\prime 2}}, \\
& T=\frac{4 \sqrt{3} a}{\sqrt{U_{1}-U_{3}}} F(\pi / 2, k)=\frac{4 \sqrt{3} a K}{\sqrt{U_{1}-U_{3}}} \\
& R=\frac{\pi K}{2 K^{\prime} T} .
\end{aligned}
$$

In Eq. (4.7), $U$ is clearly a periodic function of $z$ with period $T$, and each term in the infinite series is a soliton. This gives a representation of a periodic function by an infinite number of solitons.

It should be mentioned that

$$
c n(v, k) \approx \cos v+k^{2}[v-(\sin v)(\cos v)](\sin v) / 4
$$

when $k \ll 1[8]$. Thus under the limiting case of $k \rightarrow 0^{+}$, i e. $U_{1} \rightarrow U_{2}$, we have

$$
\begin{aligned}
U(z) & \approx U_{2}+\left(U_{1}-U_{2}\right) \cos ^{2} v \\
& =U_{2}+\frac{1}{2}\left(U_{1}-U_{2}\right)(1+\cos 2 v), \text { when } U_{1} \rightarrow U_{2} .
\end{aligned}
$$

And hence

$$
U(z) \approx A_{0}+B_{0} \cos C_{0}\left(z-z_{1}\right), \text { when } U_{1} \rightarrow U_{2},
$$

where $A_{0}=U_{2}, B_{0}=\left(U_{1}-U_{2}\right) / 2$, and $C_{0}=\sqrt{U_{1}-U_{3}} /(\sqrt{3} a)$. The function $A_{0}+B_{0} \cos C_{0}\left(z-z_{1}\right)$ is a solution for infinitesimal waves.

Under the limiting case $k \rightarrow 1^{-}$, i.e. $U_{2} \rightarrow U_{3}$, since $c n(v, k) \approx \operatorname{sech} v$, we shall obtain

$$
\begin{aligned}
U(z) & \approx U_{2}+\left(U_{1}-U_{2}\right) \operatorname{sech}^{2} v \\
& =A_{1}+B_{1} \operatorname{sech}^{2} C_{1}\left(z-z_{1}\right), \text { when } U_{2} \rightarrow U_{3},
\end{aligned}
$$

where $A_{1}=U_{2}, B_{1}=U_{1}-U_{2}$, and $C_{1}=\sqrt{U_{1}-U_{3}} /(2 \sqrt{3} a)$.

\section{CNOIDAL WAVE SOLUTION WHEN $\boldsymbol{n}=2$}

When $n=2$ we assume $A=0$ and obtain Eq. (2.4)

$$
U^{\prime 2}=\frac{1}{6 a^{2}}\left(-U^{4}+6 C U^{2}+12 B U+D\right)=\frac{1}{6 a^{2}} F(U),
$$

where $D$ is an integration constant.

Suppose that we choose the constants $B, C$, and $D$ so that the function $F(U)$ in the right-hand side of Eq. (5.1) has four distinct simple real zeros $U_{1}>U_{2}>U_{3}>U_{4}$ with $U_{4}=-U_{1}, U_{3}=-U_{2}$, and $U_{2} \leq U \leq U_{1}$. From Eq. (5.1) we can derive the cnoidal wave solution 


$$
\begin{aligned}
U(z) & =\left[U_{1}^{2}-\left(U_{1}^{2}-U_{2}^{2}\right) s n^{2}(v, k)\right]^{1 / 2} \\
& =\left[U_{2}^{2}+\left(U_{1}^{2}-U_{2}^{2}\right) c n^{2}(v, k)\right]^{1 / 2} \\
& =U_{1} d n(v, k) \\
& =U_{1} d n\left(\frac{U_{1}}{\sqrt{6} a}\left(z-z_{1}\right), k\right),
\end{aligned}
$$

where $U\left(z_{1}\right)=U_{1}, v=-\left(U_{1} /(\sqrt{6} a)\right)\left(z-z_{1}\right)$, and $k^{2}=\left(U_{1}^{2}-U_{2}^{2}\right) / U_{1}^{2}$. This solution is a periodic function with the period $T$ in $z$ given by

$$
T=2 \sqrt{6} a \int_{U_{2}}^{U_{1}} \frac{d U}{\sqrt{\left(U_{1}^{2}-U^{2}\right)\left(U^{2}-U_{2}^{2}\right)}} .
$$

Using the Fourier series expansion of $d n(v, k)$ and the Poisson's summation formula we can express the cnoidal wave solution as

$$
U(z)=Q \sum_{m=-\infty}^{\infty} \operatorname{sech} R\left(z-z_{1}+m T\right)
$$

where $Q=U_{1} \pi /\left(2 K^{\prime}\right), T=2 \sqrt{6} a K / U_{1}$, and $R=K \pi /\left(K^{\prime} T\right) \quad K$ and $K^{\prime}$ are defined following Eq. (4.6).

There are also two limiting cases of $k$ for the cnoidal wave solution given in Eq (5.2) When $k \rightarrow 0^{+}$, i e. $U_{1} \rightarrow U_{2}$, we have

$$
U(z) \approx A_{2}+B_{2} \cos C_{2}\left(z-z_{1}\right)
$$

where $A_{2}=U_{1}, B_{2}=\left(U_{1}^{2}-U_{2}^{2}\right) /\left(4 U_{1}\right)$, and $C_{2}=2 U_{1} /(\sqrt{6} a)$.

When $k \rightarrow 1^{-}$, i.e $U_{2} \rightarrow 0$, we have

$$
U(z) \approx U_{1} \operatorname{sech} C_{3}\left(z-z_{1}\right),
$$

where $C_{3}=U_{1} /(\sqrt{6} a)$

It should be mentioned that we can also obtain the cnoidal solution for Eq. (2.4) when $n=4$ [7] However the author has not been able to establish the conjecture that the cnoidal solution can also be represented by an infinite sum of solitons

\section{REFERENCES}

[1] MAXON, S and VIECELLI, J., Cylindrical solitons, Physics of Fluids, 17 (1974), 1614-1616

[2] JOHNSON, R., Water waves and Korteweg-de Vries equation, J. of Fluid Mech., 97 (1980), 701719.

[3] JOHNSON, R., The Korteweg-de Vries equation and related problems in water wave theory, Nonlinear Waves, Cambridge University Press (1983), 25-43.

[4] CHEN, Y. and WEN, S., Traveling wave solutions to the two-dimensional Korteweg-de Vries equation, J. of Math. Anal. and Appl., 127 (1987), 226-236

[5] CHEN, Y. and WEN, S., Cylindrical wave solutions to the Korteweg-de Vries equation, Arch. Mech., 44 (1992), 481-485.

[6] JOHNSON, R., On the inverse scattering transform, the cylindrical Korteweg-de Vries equation and similarity solutions, Physics Letters, 72A (1979), 197-199.

[7] CHEN, Y, WU, J. and WEN, S., An existence theorem of periodic traveling wave solutions to the power Kadomstev-Petviashvili equation, Arch. Mech., 46 (1994), 797-804

[8] BYRD, P. and FRIEDMAN, M., Handbook of Elliptic Integrals for Engineers and Scientists, 2nd ed., Springer-Verlag, New York-Berlin, 1971.

[9] OBERHATTINGER, F., Fourier Expansions, Academic Press, New York-London, 1973.

[10] COURANT, R. and HILBERT, D., Methods of Mathematical Physics, Vol. 1, Interscience Publ., 1953. 


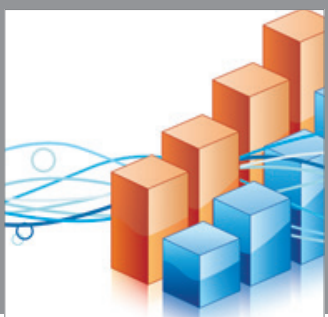

Advances in

Operations Research

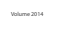

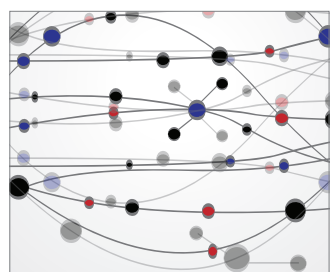

\section{The Scientific} World Journal
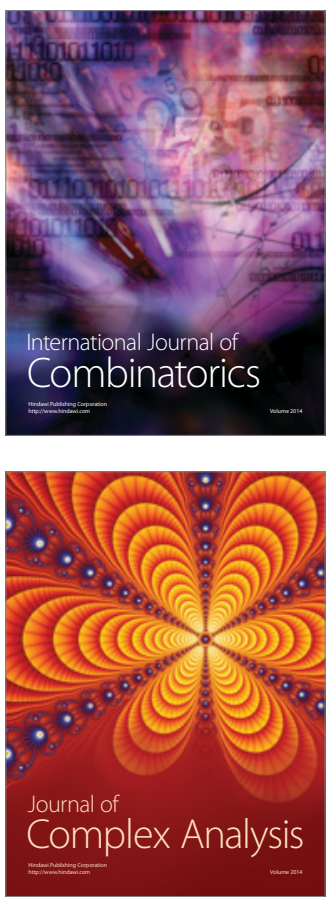

International Journal of

Mathematics and

Mathematical

Sciences
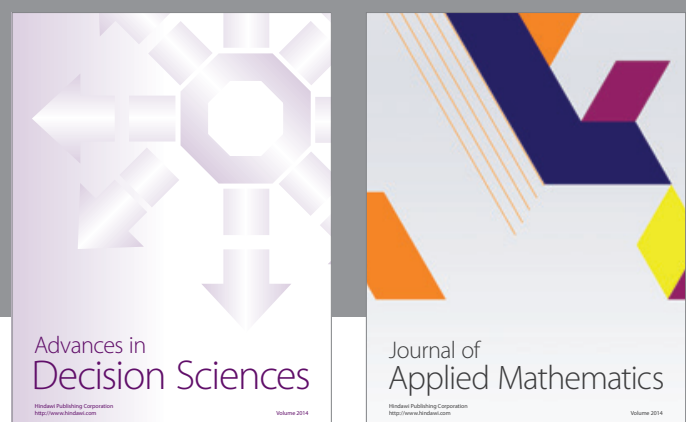

Journal of

Applied Mathematics
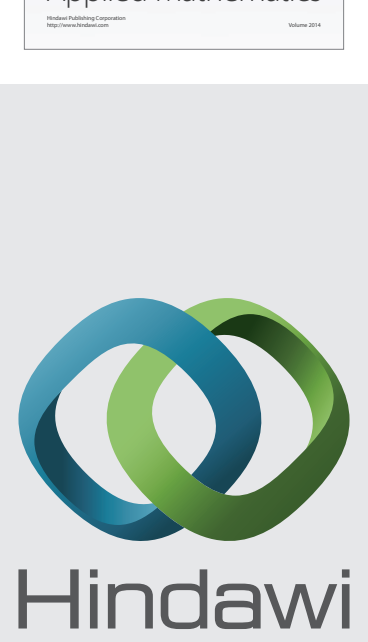

Submit your manuscripts at http://www.hindawi.com
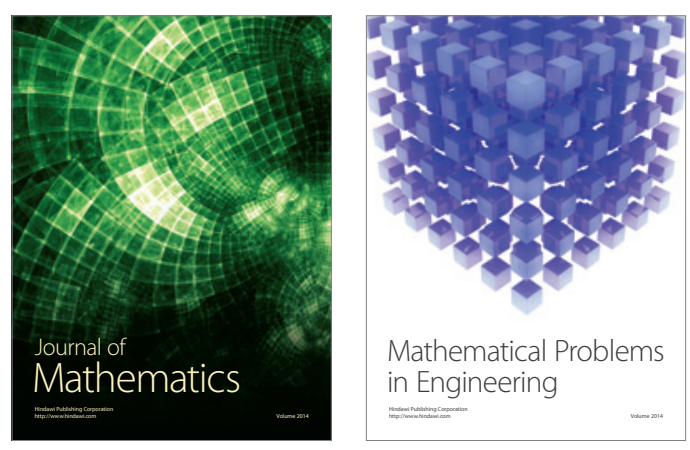

Mathematical Problems in Engineering
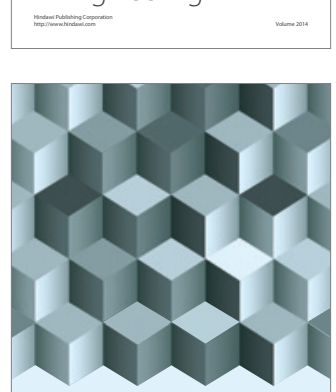

Journal of

Function Spaces
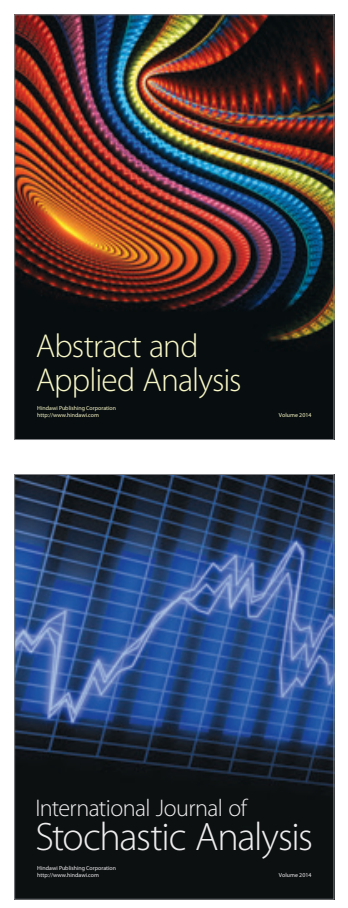

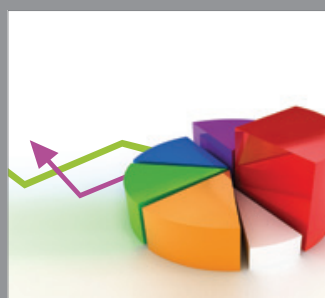

ournal of

Probability and Statistics

Promensencen
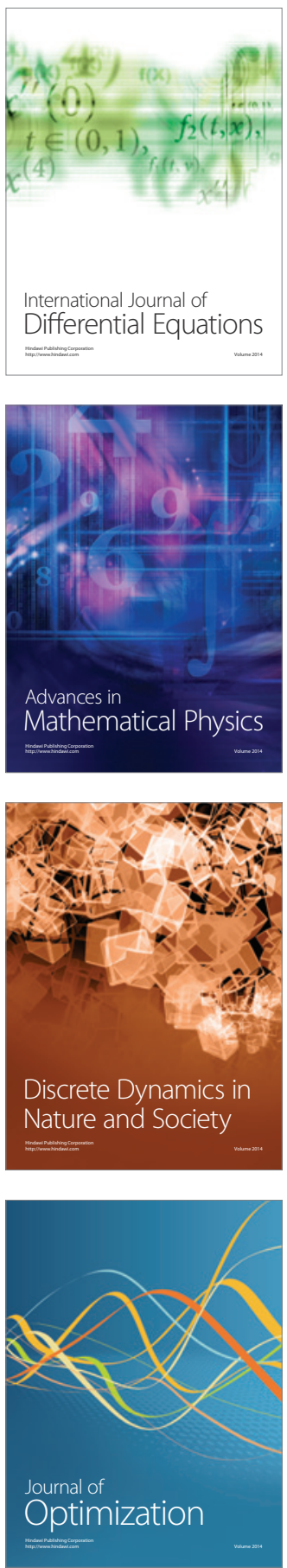